\title{
Parâmetros Petrofisicos que influenciam a propagação da onda $P$ lenta
}

\section{Francisco Cabrera Zambrano, Marco A Bassottelli Botelho, CPGG-UFBA}

Copyright 2006, SBGf - Sociedade Brasileira de Geofísica

Este texto foi preparado para a apresentação no II Simpósio de Geofísica da Sociedade Brasileira de Geofísica, Natal, 21-23 de setembro de 2006. Seu conteúdo foi revisado pela Comissão Tecno-científica do II SR-SBGf mas não necessariamente representa a opinião da SBGf ou de seus associados. E proibida a reproducão total ou parcial deste material para propósitos comerciais sem prévia autorização da SBGf.

\section{Resumo}

A teoria de Biot estabelece para um sistema poroso, saturado com um fluido viscoso, a propagação de três ondas: $\mathrm{P}$ rápida, $\mathrm{S}$ e a onda $\mathrm{P}$ lenta. $\mathrm{Em}$ particular $\mathrm{a}$ chamada onda $P$ lenta é altamente atenuada e sua propagação é análoga ao fenômeno difusivo. Neste trabalho mostra-se a influencia dos parâmetros petrofisicos descritivos de uma rocha de reservatório principalmente da viscosidade, para a resposta da onda $p$ lenta. Se resolve as equações de Biot, para valores diferentes dos parâmetros, se demonstra que para valores grandes de viscosidade, a onda $p$ lenta não é registrada.

\section{Introdução}

Nos trabalhos de Biot (1956 a,b) o resultado fundamental da sua teoria é que no meio geram-se três tipos de ondas, duas ondas $\mathrm{P}$ e uma onda $\mathrm{S}$. A primeira onda $p$ é conhecida como "onda $P$ rápida", a outra tem velocidade muito menor que a primeira; a onda $P$ lenta é altamente atenuada e sua propagação é análoga ao fenômeno de condução de calor, para freqüências baixas, o meio não pode suportar a onda $\mathrm{P}$ lenta, por ser um processo difusivo, segundo Biot (1956b) o efeito da viscosidade é dominante sobre os efeitos inerciais.

O conteúdo de fluidos no meio poroso e seu movimento no interior do meio são fatores muito importantes para avaliar por exemplo a capacidade de um reservatório ou um aqüífero. Os parâmetros fundamentais que determinam o movimento do fluido são, além da porosidade, a permeabilidade e a viscosidade, os quais são os responsáveis da atenuação da onda $P$ lenta .

Define-se $\quad e=\operatorname{div} \overrightarrow{\mathrm{u}} \in=\operatorname{div} \overrightarrow{\mathrm{U}}$, onde $u$ e $U$ são respectivamente os deslocamentos do sólido e do fluido. As equações seguintes descrevem a propagação sísmica em um meio poroso e homogêneo

$$
\begin{aligned}
& N \nabla^{2} \overrightarrow{\mathrm{u}}+(A+N) \nabla e+Q \nabla \in=\frac{\partial^{2}}{\partial t^{2}}\left(\rho_{11} \overrightarrow{\mathrm{u}}+\rho_{12} \overrightarrow{\mathrm{U}}\right)+b \frac{\partial}{\partial t}(\overrightarrow{\mathrm{u}}-\overrightarrow{\mathrm{U}}) \\
& \nabla(\mathrm{Q} \mathrm{e}+\mathrm{R} \in)=\frac{\partial^{2}}{\partial t^{2}}\left(\rho_{11} \overrightarrow{\mathrm{u}}+\rho_{12} \overrightarrow{\mathrm{U}}\right)-b \frac{\partial}{\partial t}(\overrightarrow{\mathrm{u}}-\overrightarrow{\mathrm{U}}) .
\end{aligned}
$$

Se o meio é estatisticamente isotrópico, a onda $s$ é desacoplada das ondas compressionais $p$. Tomando a divergência nas equações anteriores temos a versão acústica das equações de Biot:

$$
\begin{aligned}
& \nabla^{2}(P e+Q \in)=\frac{\partial^{2}}{\partial t^{2}}\left(\rho_{11} e+\rho_{12} \in\right)+b \frac{\partial}{\partial t}(e-\epsilon) \\
& \nabla^{2}(Q e+R \in)=\frac{\partial^{2}}{\partial t^{2}}\left(\rho_{12} e+\rho_{22} \in\right)-b \frac{\partial}{\partial t}(e-\epsilon) .
\end{aligned}
$$

Os parâmetros $P, Q, R$ são coeficientes elásticos e os coeficientes de massas $\rho$ são escritos em termos dos módulos de compressibilidade do fluido $\mathrm{K}_{\mathrm{f}}$, o modulo de compressibilidade do sólido $\mathrm{K}_{\mathrm{s}}$, o modulo da matriz $\mathrm{K}_{\mathrm{m}} \mathrm{e}$ os parâmetros de porosidade $\phi$, viscosidade $\eta \mathrm{e}$ permeabilidade $\kappa$.

$$
\begin{aligned}
& \rho_{11}=\rho_{s}(1-\phi)+\phi \rho_{f}(\tau-1) \\
& \rho_{12}=\phi \rho_{f}(1-\tau) \\
& \rho_{22}=\tau \phi \rho_{f} \\
& P=\left[(1-\phi)(\alpha-\phi) K_{s}+\phi K_{s} K_{m} / \mathrm{K}_{\mathrm{f}}\right] / \mathrm{D}+4 \mu / 3 \\
& \mathrm{R}=\phi^{2} K_{s} / \mathrm{D} \\
& \mathrm{Q}=(\alpha-\phi) \phi \mathrm{K}_{\mathrm{s}} / \mathrm{D} \\
& \mathrm{D}=\alpha-\phi+\mathrm{K}_{\mathrm{s}} / \mathrm{K}_{\mathrm{f}} \\
& \alpha=1-K_{m} / \mathrm{K}_{\mathrm{s}} \\
& b=\eta \phi^{2} / \kappa,
\end{aligned}
$$

Onde a é uma variável intermediaria e $\tau$ é chamada de tortuosidade. O termo $b$ é fundamental para obter $a$ segunda onda $\mathrm{P}$ e está relacionado com os três parâmetros fundamentais: porosidade, viscosidade e permeabilidade; se fosse zero, a onda p lenta apareceria como se fosse a onda $\mathrm{P}$ rápida, no caso de que a viscosidade aumenta, a atenuação da onda é muito rápida e não é possível ter registro dela nos pontos mais afastados da fonte; além disso, mudando os valores dos parâmetros se obtém diferentes respostas do meio, desde que leve-se em conta a freqüência $\omega$ da fonte que excita o meio.

Nos trabalhos de Biot (1956b, 1962a,b) o termo de atenuação $b$ é função da freqüência; definindo o chamado operador viscoelastico, o qual é um operador que descreve a combinação dos efeitos inerciais e de fricção, desta forma Biot obtém seus resultados em todo o espectro de freqüências. Carcione (1996) fornece soluções analíticas no caso acústico para o espectro das freqüências baixas e altas, ele mostra que os fatores 
principais na resposta sísmica são a porosidade, a permeabilidade e a viscosidade.

A existência da segunda onda compressional, depois de que Biot (1956a, 1956b, 1962) predisse, foi sujeita a muitas especulações durante vários anos. Mais de uma década depois se teve evidencia de sua existência, em condições experimentais apropriadas.

\section{Metodologia/ Problema Investigado}

Neste trabalho se resolve as equações de Biot no caso do meio poroso homogêneo usando um modelo $2 \mathrm{D}$ de comprimento $2250 \mathrm{~m}$ em x e $z$, vide figura 1 .

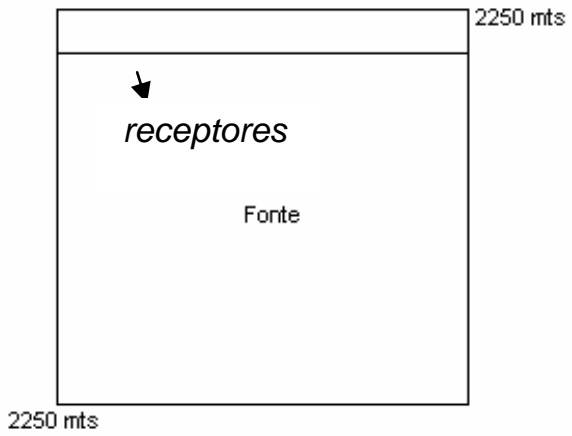

Figura 1 Descrição geométrica do arranjo fonte- receptores.

Coloca-se uma fonte pontual no centro $(1125,1125)$. A expressão funcional da fonte é $F(x, z, t)=\delta(x, z) f(t)$ onde $\mathrm{f}(\mathrm{t})$ e a derivada da gaussiana. Como o meio é bifásico a fonte é distribuída nas partes sólidas e fluidas: $\mathrm{f}_{\mathrm{s}}=(1-\phi) \mathrm{F}$, $\mathrm{f}_{\mathrm{f}}=\phi \mathrm{F}$, com sua intensidade ponderada pela porosidade. Vide os trabalhos de Hassanzadeh (1991) e de Pinheiro e Botelho (1996). Os receptores são colocados na profundidade de $z=264 \mathrm{~m}$.

As equações são resolvidas por meio de um esquema de diferenças finitas de segundo ordem no tempo e de quarta ordem no espaço, para diminuir a dispersão numérica se tomou um passo de malha de $10 \mathrm{mts}$ nas direções $x$ e $z$. Foram feitas simulações para diferentes valores dos parâmetros $\phi, \eta$ e $\kappa$. Para resolver as equações de Biot, se faz necessário escrever as equações na forma matricial, definindo as seguintes matrizes:

$$
\begin{aligned}
& M=\left(\begin{array}{ll}
\rho_{11} & \rho_{12} \\
\rho_{12} & \rho_{22}
\end{array}\right) \quad T=\left(\begin{array}{ll}
P & Q \\
Q & R
\end{array}\right) \text { a matriz de atenuação } \\
& B=\left[\begin{array}{cc}
b & -b \\
-b & b
\end{array}\right] \text { e vetor } Z=\left[\begin{array}{l}
e \\
\in
\end{array}\right],
\end{aligned}
$$

então o sistema de Biot fica da seguinte forma :

$$
T \nabla^{2} Z=B \dot{Z}+M \ddot{Z}
$$

Na esquerda estão as derivadas espaciais e na direita as derivadas temporais. Seja $\Omega^{n}$ o valor dessa variável no tempo $n$, então definimos as variáveis: $F^{n}$ e $G^{n}$ dadas por:

$\left[\begin{array}{l}F^{n} \\ G^{n}\end{array}\right]=\left[\Delta t^{2} T \nabla^{2}+2 M\right] Z^{n}-[M-0.5 \Delta t B] Z^{n-1}$.

O laplaciano é calculado com um esquema de diferenças finitas de quarta ordem, embora possa ser calculado utilizando um método pseudo espectral. Definindo os seguintes valores

$$
\begin{aligned}
& a_{11}=\rho_{11}+0.5 b \Delta t \\
& a_{12}=\rho_{12}-0.5 b \Delta t \\
& a_{22}=\rho_{22}+0.5 b \Delta t \\
& \text { det }=a_{11} a_{22}-a_{12} a_{12} .
\end{aligned}
$$

Então os valores das variáveis $e, \in$ no tempo $n+1$ é dada por:

$$
\begin{aligned}
& e^{n+1}=\left[F^{n} a_{22}-G^{n} a_{12}\right] / \mathrm{det} \\
& \epsilon^{n+1}=\left[G^{n} a_{11}-F^{n} a_{12} / \mathrm{det}^{\circ} .\right.
\end{aligned}
$$

A escolha do passo de tempo $d t$ se baseia no critério de estabilidade: $d t \leq \frac{d x}{\sqrt{2} V_{p}} \quad, d x$ o passo da malha na direção $x(d x=d z)$ e $V_{p}$ a velocidade da onda $\mathrm{P}$ rápida. $\mathrm{O}$ esquema de propagação é similar ao proposto por Hassanzadeh (1991). As equações acústicas de Biot podem ser desacopladas em alguns casos, por exemplo supondo viscosidade zero e quando os parâmetros $\rho_{12}$ e $Q$ são identicamente zero, neste caso temos duas ondas independentes representadas pelas equações:

$$
\begin{aligned}
& \nabla^{2} e=\frac{\rho_{11}}{P} \ddot{e} \\
& \nabla^{2} \in=\frac{\rho_{22}}{R} \ddot{\epsilon} .
\end{aligned}
$$

Pelas expressões destes parâmetros em termos dos parâmetros petrofisicos, se tem que $\rho_{12}$ é zero quando $\tau$ a tortuosidade é 1 e $Q$ é zero se $K_{m}=K_{s}(1-\phi)$ e por tanto as equações anteriores ficam:

$$
\begin{aligned}
& \nabla^{2} e=\frac{\rho_{s}}{K_{s}} \ddot{e} \\
& \nabla^{2} \in=\frac{\rho_{f}}{K_{f}} \ddot{\epsilon} .
\end{aligned}
$$

Essas equações significam duas ondas $P$ totalmente independentes; uma no sólido e outra no fluido. No caso de não ter desacoplamento as duas ondas correspondem ao movimento em fase do sólido e fluido para a onda $P$ rápida e ao movimento fora de fase para a onda $\mathrm{P}$ lenta.

Os valores dos parâmetros utilizados no experimento mostrado neste trabalho são fornecidos abaixo: 
$K_{\mathrm{s}}=35 \times 10^{9} \mathrm{~Pa}$

$K_{m}=1.7 \times 10^{9} \mathrm{~Pa}$

$K_{f}=2.4 \times 10^{9} \mathrm{~Pa}$

$\phi=0.3$ Porosidade

$\tau=2 \quad$ Tortuosidade

$\rho_{\mathrm{s}}=2650 \mathrm{~kg} / \mathrm{m}^{3}$ Densidade do solido

$\rho_{f}=1000 \mathrm{~kg} / \mathrm{m}^{3} \quad$ Densidade do fluido

$\eta \leq 0.1$ cp Viscosidade do fluido

$\kappa=1 \times 10^{-12} \quad$ Permeabilidade hidraulica .

\section{Resultados}

A seguir vamos mostrar os resultados numéricos obtidos para modelo acima descrito. Nos diferentes casos, as velocidades das ondas $P$ rápida e lenta são em meia de $3634 \mathrm{~m} / \mathrm{seg}$ e $1540 \mathrm{~m} / \mathrm{seg}$ respectivamente, tomandose uma porosidade $\phi$ igual a 0.3 . Nas Figuras 1 e 2 mostra se instantâneas das frentes de ondas produzidas no instante de tempo de $0.16 \mathrm{~s}$ e $0.33 \mathrm{~s}$, nos casos de viscosidade zero (figura a) e viscosidade diferente de zero (figura b) . Como é esperado a onda $P$ lenta é visualizada (registrada) melhor no caso de atenuação nula, embora sua amplitude seja sempre menor que a amplitude da onda $P$ rápida.

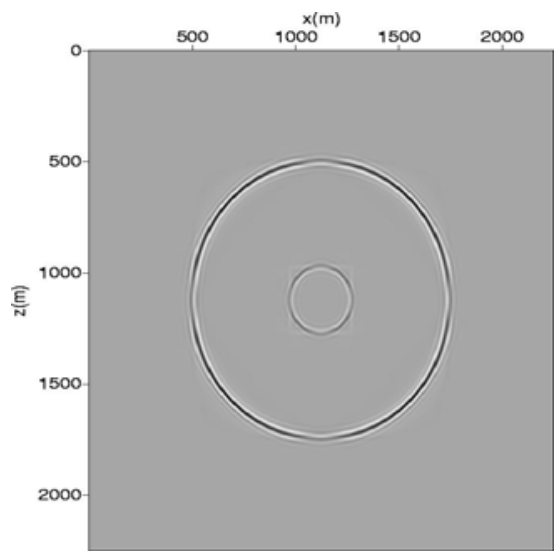

(a)

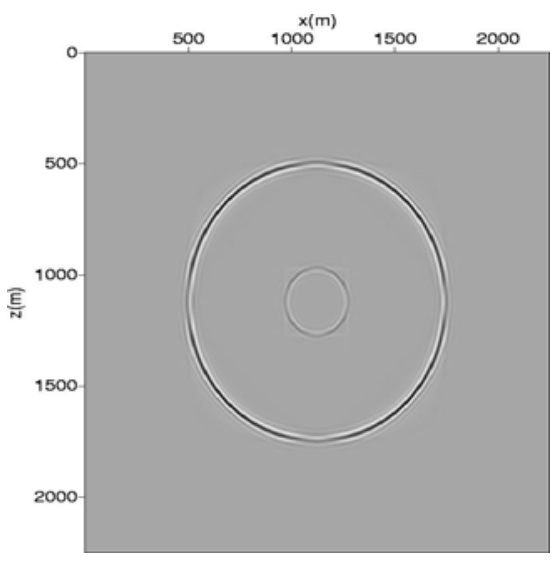

(b)

Figura $1 \mathrm{a}-b$. As duas ondas $P$ no tempo $t=0.16 \mathrm{~s}$ (a) $\eta=0$, (b) $\eta=1.0 \times 10^{-2} c p$

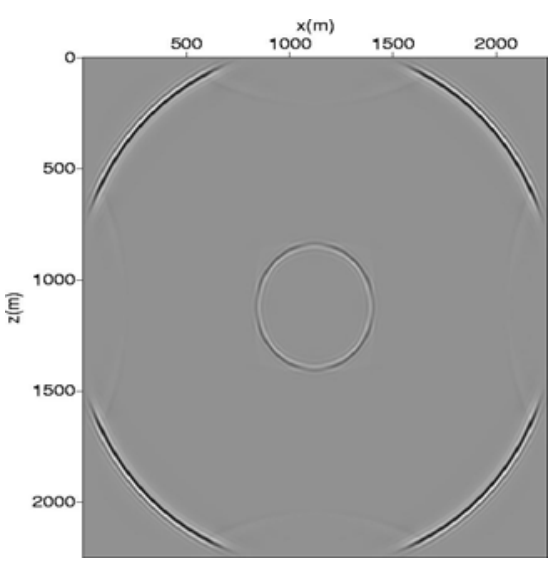

(a)

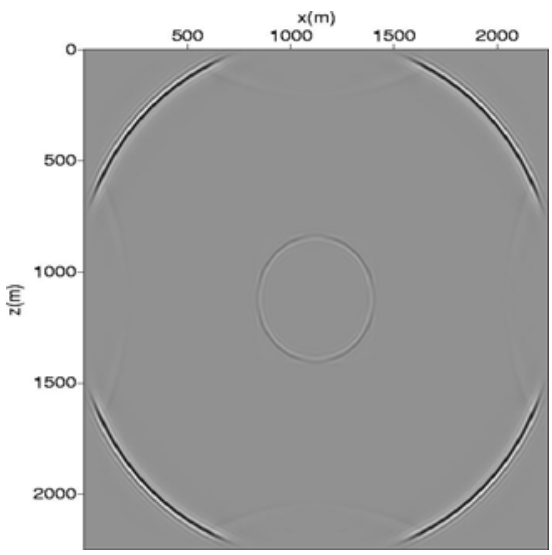

(b)

Figura $2 \mathrm{a}-\mathrm{b}$. As duas ondas $p$ no tempo $t=0.33 \mathrm{~s}$ (a) $\eta=0$, (b) $\eta=1.0 \times 10^{-2} \mathrm{cp}$

Pode ver que a onda $\mathrm{P}$ lenta é atenuada rapidamente e sua visualização (registro) é mais difícil para tempos maiores. Comparando as Figuras 3 e 4 que mostram só a onda P lenta no tempo $0.4 \mathrm{~s}$ nos casos de viscosidade nula e viscosidade não nula. Existem os artefatos da dispersão numérica e principalmente reflexões de borda da onda $P$ rápida que foi mal absorvida pelas bordas absorcivas. Vide figura 3 e 4.

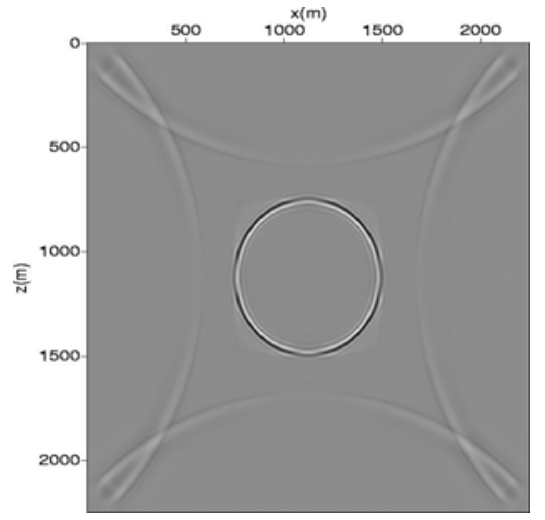

Figura 3. A onda $p$ lenta no tempo $t=0.4 \mathrm{~s}$ $\eta=0 \mathrm{cp}$. 


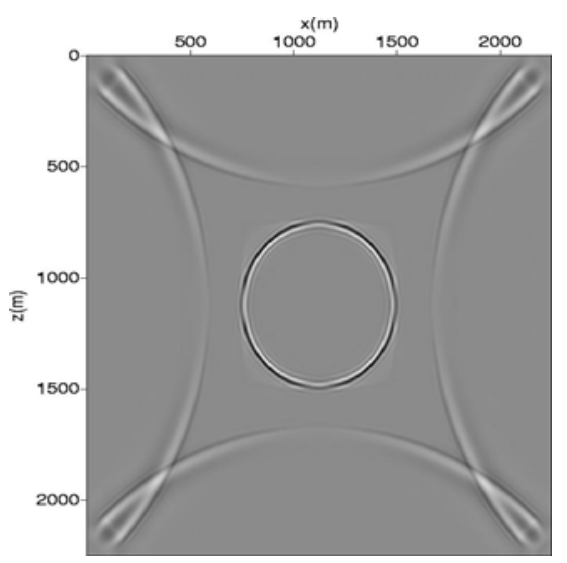

Figura 4. A onda $p$ lenta no tempo $t=0.4 \mathrm{~s}$ $\eta=1.0 \times 10^{-2} \mathrm{cp}$.

Finalmente apresenta-se um sismograma para um tempo de registro de 1.3 segundos; onde o sismograma foi construído considerando-se o caso irreal da viscosidade nula para que a onda $P$ lenta fique bem definida.

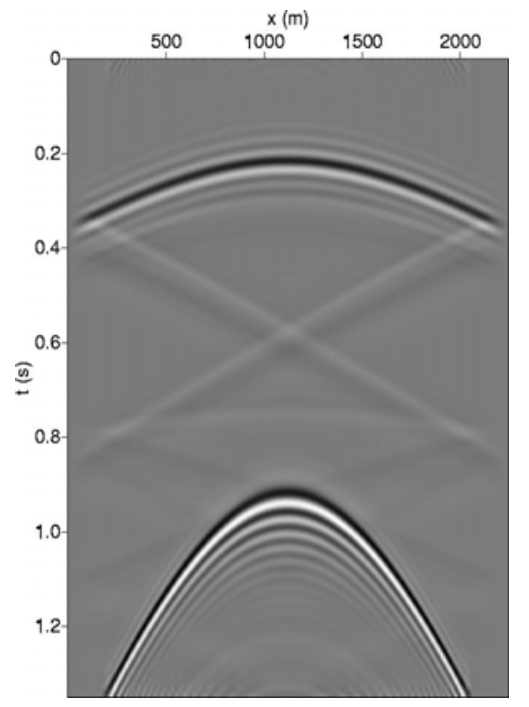

Figura 5. Sismograma no caso de viscosidade zero.

\section{Conclusões}

Neste trabalho mostra-se a influência dos valores dos parâmetros petrofísicos, na propagação da onda p lenta. Os parâmetros fundamentais são a viscosidade, a porosidade e permeabilidade hidráulica, observa-se que quanto maior for a viscosidade, o termo b correspondente à atenuação será maior e por tanto a onda $p$ lenta será mais difícil de visualizar, acentuando o caráter difusivo desta onda. O valor empregado da porosidade para os resultados mostrados foi de 0.3 , de $1 . \times 10^{-12}$ para a permeabilidade e a viscosidade no intervalo de zero ate um valor maximo de $3 \times 10^{-4}$.
Nesta pesquisa utilizou-se uma malha quadrada simples e emprego-se operadores de quarta ordem para resolver as derivada espaciais e de segunda ordem para resolver as derivadas temporais.

O fenômeno da dispersão numérica torna mais difícil a captação da onda $\mathrm{P}$ lenta, para tempos maiores $\mathrm{e}$ posições afastadas da fonte, devido a isso os autores propõem resolver as equações utilizando outros esquemas como as malhas diferenciadas e resolver as derivadas espaciais com os métodos pseudo espectrais.

\section{Agradecimentos}

O primeiro autor agradece a CAPES pelo suporte financeiro através da bolsa de estudos. $O$ segundo autor agradece a Rede de Risco exploratório, CTPETRO/ CNPQ- FINEP pelo apoio estructural.

\section{Referencias}

Biot M.A .1956. Theory of propagation of elastic waves in a fluid -saturaded porous solid. I. Low -frequency range. Journal of the Acoustical Society of America 28,168-178.

Biot M.A .1956. Theory of propagation of elastic waves in a fluid -saturaded porous solid. I. Higher -frequency range. Journal of the Acoustical Society of America 28,179-191.

Biot M.A .1962. Mechanic of deformation and acoustic propagation in porous media. Journal of Applied Physics 33,1482-1498.

Biot M.A. 1962. Generalized theory of acoustic propagation in porous dissipative media. Journal of the Acoustical Society of America 34, 1254-1264.

Carcione J.M. 1996. Full frequency-range transient solution for compressional waves in a fluid satured viscoacoustic porous medium. Geophysical Prospecting 44, 99-129.

Hassanzadeh S. 1991. Acoustic modeling in fluid- satured porous medium. Geophysics 56, 424-435.

Pinheiro N, V., e Botelho, MAB., 1996, Solução do sistema de equações de Biot para meios acústicos porosos usando diferenças finitas com operadores de quarta ordem, Revista Brasileira de Geofísica 14(2) 131145. 